\title{
THE USE OF METEOR RADARS IN THE DETECTION OF INFALLING SPACE DEBRIS
}

\author{
D.I.OLSSON-STEEL
}

Department of Physics and Mathematical Physics, University of Adelaide, G.P.O.Box 498, Adelaide, SA 5001, Australia.

\section{W.G.ELFORD}

Spaceguard Orbital Debris Analysts, 51 Warwick Street, Enfield, SA 5085, Australia.

\begin{abstract}
Radars of various configurations are used to detect the flux of meteors into the atmosphere, and therefore may be of use in characterizing the artificial meteors produced by small $(100$ microns $-1 \mathrm{~cm})$ pieces of space debris upon re-entry. Both conventional and over-the-horizon radars are discussed here, and the ways in which they may be deployed in order to detect infalling space debris are outlined.
\end{abstract}

\section{INTRODUCTION}

Small particles of space debris (masses below a microgram) can be detected via impacts on sensors or exposed surfaces in Earth-orbit (e.g. Laurance and Brownlee, 1986). However, these techniques have the drawbacks of (i) lack of temporal resolution; (ii) lack of trajectory information; and also (iii) inability to detect a statistical sample of larger particles due to the small area of the detector. Larger particles (meteoroids) in the size range 100 microns $-10 \mathrm{~cm}$ (masses $10^{-6}$. $10^{3} \mathrm{gm}$ ) are detectable through radar/optical meteor techniques, which means that the natural influx can be monitored, as well as re-entering debris.

The space debris in this size range poses the major threat to operational satellites, but is not readily detectable in space (e.g. Kessler, 1985). Thus it is of considerable importance to be able to detect such 'artificial meteors' as the debris re-enters the atmosphere. Obviously such detections represent particles which have already undergone orbital decay, but nevertheless a knowledge of the influx of these objects should allow one to work back to the flux and orbital distribution in space, and thus check on models of the cloud structure of such small pieces of debris. It is the aim of this paper to briefly outline how meteor radars may be used to detect infalling debris, and to differentiate between the natural and manmade flux. 


\section{CONVENTIONAL METEOR RADARS}

Typically, meteor radars have been operated in the frequency range 20-70 $\mathrm{MHz}$ (i.e. in the VHF region of the radio spectrum), with the antenna beams directed well away from overhead, at zenith angles from $30^{\circ}$ to $80^{\circ}$ (e.g. see McKinley, 1961, and Hughes, 1978). Echoes from ranges $150-1000 \mathrm{~km}$ are then gated and the meteor range and echo amplitude and duration etc. are recorded. The reason for the use of high zenith angles is that the natural meteoroids, with radiants spread over the celestial sphere, are more readily detected with this geometry as a larger volume of the atmosphere acts as a collector. For typical meteor radars count rates of 10 or more per minute are attainable.

The heights of meteors detected by VHF radars have mostly been in the 85$105 \mathrm{~km}$ range, with individual heights being dependent upon the velocity, structure and trajectory of the meteoroid. However, recent work with HF radars (at frequencies of 2 and $6 \mathrm{MHz}$ ) have shown that in fact the majority of natural meteoroids ablate higher than this, at $110-120 \mathrm{~km}$, with some meteors being detectable to at least $140 \mathrm{~km}$ (Olsson-Steel and Elford, 1987a,b; Thomas et al, 1988; Elford and Olsson-Steel, 1988). In the present connection these 'highaltitude' meteors are not of great significance since the relatively high-density, low-velocity space debris will ablate at lower altitudes, below the height limit for VHF radars (see Olsson-Steel, 1988, for a discussion of the variation of meteor height with bulk density).

A meteor resulting from an inspiralling item of space debris will have a radiant close to the horizon; the meteor trail will be almost horizontal. The specular-reflection condition of a backscatter meteor echo (see McKinley, 1961) thus requires that the antenna beam be pointed vertically. The beam must also be narrow and have weak side-lobes else natural meteoroids from radiants well above the horizon will dominate the count-rate. Even then such a system has two distinctive drawbacks:

(i) The influx of natural meteoroids (about 50 tonnes per day: Thomas et al, 1988 ) is much higher than the debris influx. Thus some thought will need to be given to how to distinguish between the two sources of ionized trails. Possible criteria are: velocity measurements; altitude measurements (the debris being of higher density would be expected to give trails at lower altitudes); accurate determinations of radiants; and measurements of the diurnal variation in the count rate.

(ii) A vertically-pointed radar illuminates a very small volume of the atmosphere so that the count rate (for all meteors) is much lower than for a conventional meteor radar with the beam directed at high zenith angles.

Despite these drawbacks, this type of radar should be capable of supplying invaluable information regarding the flux and ablation characteristics of smaller debris particles of a size $(100$ microns $-1 \mathrm{~cm})$ not accessible in other ways. A dedicated radar is now under construction in Houston (Kessler, personal communication, 1988), and installations elsewhere which were designed as loweratmosphere radars ('wind profilers') may also be programmed for such use. For example, the $54 \mathrm{MHz}$ radar at Adelaide (South Australia) has already been extensively tested as a meteor detector (Olsson-Steel and Elford, 1987b; OlssonSteel, 1988), and has a narrow beam steerable between zenith angles of $0^{\circ}$ and 41' ; the Poker Flat (Alaska) and Platteville (Colorado) radars have also been used as detectors of natural meteors (Wang et al, 1988). 


\section{OVER-THE-HORIZON RADARS AS DEBRIS-METEOR DETECTORS}

An over-the-horizon (OTH) radar uses forward-scatter from the F-region in order to obtain echoes from targets which are not directly visible from the radar site due to the Earth's curvature. The beam is thus directed to very large zenith angles, just above the horizon. Although this might at first appear to contradict the requirements for a radar usable in debris-detection as discussed in section 2, in fact this is not the case. In this section the way in which such an OTH radar might be used to this end is discussed.

A fairly-typical OTH radar is the Jindalee installation in central Australia, which has been used by Thomas et al $(1986,1988)$ for the detection of echoes of meteoric origin; in fact, we believe this to be the most powerful meteor detector ever utilized, with the limiting mass being below a microgram and close to the micrometeor limit. The Jindalee radar operates at frequencies in the range 6 to $30 \mathrm{MHz}$, and has high-gain (highly-directional) antennas.

Using the formalism of Elford (1964), Thomas et al (1988) have calculated the so-called 'Response Function' for a model OTH radar. This function $\mathrm{n}(\mathrm{z}, \mathrm{A})$ is a measure of the relative response of the system to a meteor with radiant at zenith angle $z$ and azimuth $A$; the full expression for $n(z, A)$ is quite complicated and requires a numerical integration over all heights (ranges and elevation angles) and all azimuths within the antenna beam, and will not be repeated here due to space constraints. Thomas et al (1988) have shown that $\mathrm{n}(\mathrm{z}, \mathrm{A})$ peaks at high values for the zenith angle for various modes of transmission (i.e. various ray paths). Here we will consider just the simplest: direct line-ofsight backscatter, as occurs in conventional meteor radars.

In this case the zenith angle of the radiant $(\mathrm{z})$ enters into the calculation in two main places:

(i) There is a term of the form $(\operatorname{cosec} z)^{c}$, where $c$ is the electron line density exponent index, and the value of $\mathrm{c}$ found for natural meteoroids is about -1 . Thus this term becomes $\sin \mathrm{z}$, so that $\mathrm{n}(\mathrm{z}, \mathrm{A})$ increases with the radiant zenith angle, $\mathrm{z}$.

(ii) The normalized range of the echo is:

$$
r=\left(R / R_{E}\right)=\left[\sin ^{2} \text { theta }+\left(2 h / R_{E}\right]^{1 / 2}-\sin \right. \text { theta }
$$

where $R$ is the range, $R_{E}$ is the radius of the Earth, $h$ is the meteor height, and theta is the elevation angle of the meteor echo; theta is related to $\mathrm{z}$ by:

$$
\sin \text { theta }=\cos \mathrm{z} \cos \mathrm{phi}
$$

where phi is the azimuthal angle measured from the center of the antenna beam, so that:

$$
\sin (\mathrm{psi}-\mathrm{A}-\mathrm{pi})=\sin \text { phi } \sec \text { theta }
$$

where psi is the true azimuth of the echo point measured from the radar site. Since meteors occur within a limited span of heights, theta is restricted for any particular range and hence meteors of particular radiant zenith angles are selected out. 
In Figure 1, we show for a model radar the dependence of the Response Function on $\mathrm{z}$ only (the azimuth $\mathrm{A}$ is set to zero) for meteors at heights of 90 and $120 \mathrm{~km}$; the range is limited to $250<\mathrm{R}<750 \mathrm{~km}$, and the gains of the transmitting and receiving antennas have the form:

$$
\begin{array}{lr}
G_{T}, G_{R}=(\text { constant }) \cos (2 \text { theta }) & \text { phi }<10^{\circ} \\
G_{T}, G_{R}=0 & \text { phi }>10^{\circ}
\end{array}
$$

i.e. a beam narrow in azimuth which drops off rapidly with increasing elevation. As can be seen, $\mathrm{n}(\mathrm{z})$ is height-dependent, and no meteors with $\mathrm{z}<60^{\circ}$ or $\mathrm{z}$ $>80^{\circ}$ are detectable.

Since there would still be appreciable numbers of natural meteoroids with such radiants, it is necessary to restrict $\mathrm{z}$ further. To illustrate how this can be achieved very simply, Figure 2 shows $n(z)$ calculated in the same way as for Figure 1 , but now with $750<\mathrm{R}<1000 \mathrm{~km}$; clearly it is possible to very precisely choose the window of radiant zenith angles required, and thus favor debris-meteors as opposed to natural meteors. Even better selection is possible using the other modes of an OTH radar, (e.g. transmitter - F-region - forward-scatter from meteor trail - reflection from ground - F-region - receiver) and it is suggested that this may be a suitable technique for the isolation of debris-derived meteor echoes.

\section{SUMMARY}

The smaller items of space debris in circum-terrestrial orbit, which pose the greatest threat to safe operations in that region, cannot be easily tracked in space but are detectable by radar as a result of the ionization trails (artificial meteors) which they produce upon re-entry, thus allowing their essential characteristics (flux, trajectories) to be modelled. However, it is difficult to distinguish between natural and artificial meteors, and the influx of the former is several orders of magnitude higher than that of the latter. Thus, new techniques are needed which allow the two to be differentiated. Here we have discussed in general terms both conventional meteor radars and OTH radars, and suggested how each may be utilized in attacking this problem.

\section{REFERENCES}

Elford, W.G., 1964. "Calculation of the Response Function of the Harvard Radio Meteor Project Radar System", Harvard Radio Meteor Project Research Report Number 8, Cambridge, Mass.

Elford, W.G. and Olsson-Steel, D., 1987a. "The height distribution of radar meteors: Observations at $6 \mathrm{MHz}$ ", J. Atmos. Terr. Phys. (in press).

Hughes, D.W., 1978. "Meteors", pp.123-185 in "Cosmic Dust" (ed. J.A.M.McDonnell), Wiley, Chichester.

Kessler, D.J., 1985. "Orbital Debris Issues", Adv. Space Res., 5, 3-10.

Laurance, M.R. and Brownlee, D.E., 1986. "The flux of meteoroids and orbital space debris striking satellites in low Earth orbit", Nature, 323, 136-138.

McKinley, D.W.R., 1961. "Meteor Science and Engineering", McGraw-Hill, New 
York.

Olsson-Steel, D. and Elford, W.G., 1987a. "The height distribution of radar meteors: Observations at 2 MHz", J. Atmos. Terr. Phys., 49, 243-258.

Olsson-Steel, D. and Elford, W.G., 1987b. "The true height distribution and flux of radar meteors", Publ. Astron. Inst. Czechoslov. Acad. Sci., No.67, Vol.2, 193-197.

Olsson-Steel, D., 1988. "The origin and physical characteristics of meteoroids", Highlights of Astronomy (in press).

Thomas, R.M., Whitham, P.S. and Elford, W.G., 1986. "Frequency dependence of radio meteor echo rates", Proc. Astron. Soc. Australia, 6, 303-306.

Thomas, R.M., Whitham, P.S. and Elford, W.G., 1988. "Response of High Frequency radar to meteor backscatter", J. Atmos. Terr. Phys. (in press).

Wang, S.T., Tetenbaum, D., Balsley, B.B. Obert, R.L., Avery, S.K. and Avery, J.P., 1988. "A meteor echo detection and collection system for use on VHF radars", Radio Sci., 23, 46-54. 

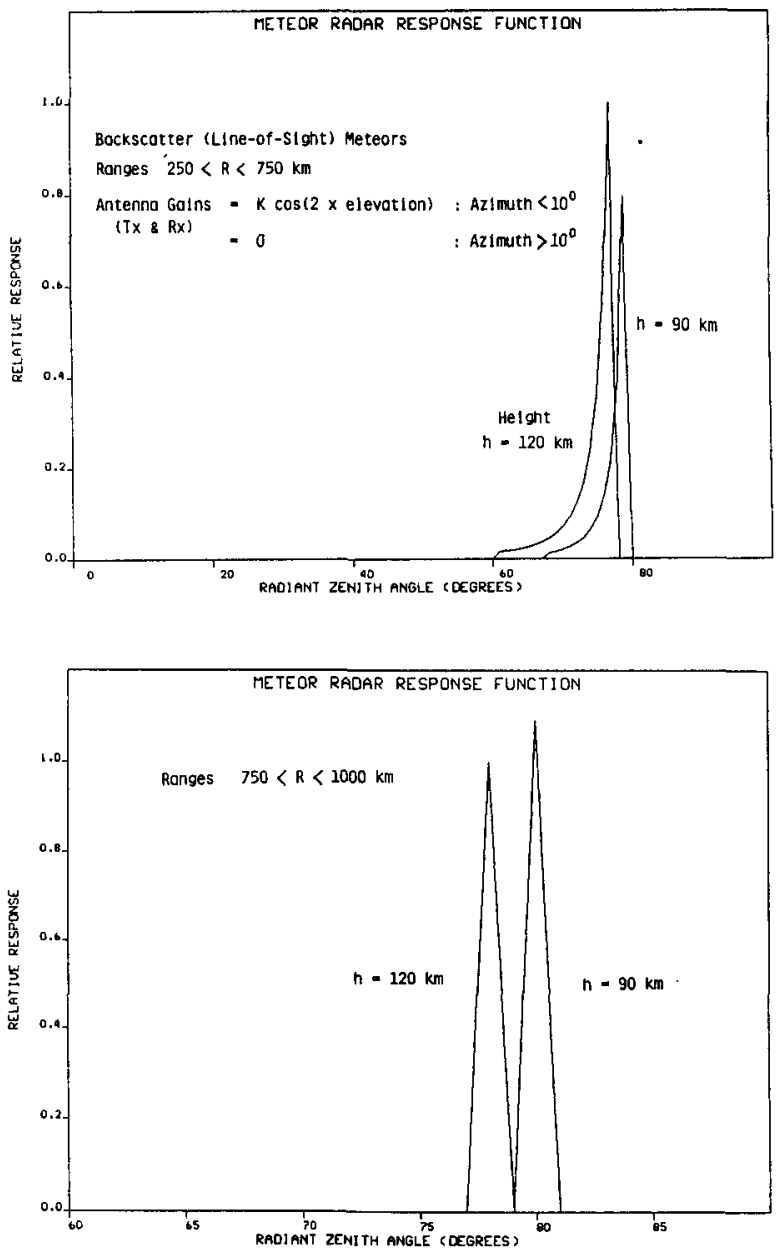

Figure 1. The response of a model radar to meteor backscatter as a function of the radiant zenith angle for two different meteor heights; the antenna gains used are as given, and only ranges from 250 to $750 \mathrm{~km}$ are included.

Figure 2 As for Fig.1 but including only meteor ranges from 750 to $1000 \mathrm{~km}$; now even higher radiant zenith angles (Le. as required for debris detection) are selected. 